\title{
Les Acteurs Du Transport Des Produits Vivriers Au Marche De Gros De Bouake (Côte d'Ivoire)
}

\author{
Bamba Vakaramoko \\ Doctorant, Institut de Géographie Tropicale, \\ Université Félix Houphouët Boigny, Abidjan, Côte d'Ivoire \\ Koffié-Bikpo Céline Yolande \\ Professeur Titulaire, Institut de Géographie Tropicale, \\ Université Félix Houphouët Boigny, Abidjan, Côte d'Ivoire
}

Doi:10.19044/esj.2019.v15n2p169～ＵRL:http://dx.doi.org/10.19044/esj.2019.v15n2p169

\section{Resume}

La chaîne d'approvisionnement des produits vivriers au marché de gros de Bouaké présente des particularités propres aux grandes villes africaines, celle d'avoir plusieurs acteurs sur toute la chaîne, de la production à la commercialisation en passant par l'acheminement et la redistribution des produits. Le rôle joué par ces acteurs est fondamental dans l'approvisionnement des localités en vivre. La présente étude vise à identifier les acteurs du transport et à analyser leur rôle dans l'acheminement des produits vivriers au marché de gros de Bouaké. La recherche documentaire, la collecte des données, leur traitement et l'analyse des résultats constituent les principales articulations de la démarche méthodologique adoptée. Pour ce qui est de la connaissance des acteurs du transport au marché de gros de Bouaké, l'étude a utilisé l'approche des enquêtes de surveillance de comportement pour analyser le rôle des acteurs dans le ravitaillement du marché de gros sur la période du 23 avril 2018 au 05 mai 2018. Ainsi, 375 transporteurs ont été enquêtés. Les résultats obtenus montrent deux types d'acteurs : les acteurs étatiques du transport des produits vivriers et les acteurs non étatiques. Cette étude met donc en évidence l'importance des acteurs du transport dans l'acheminement des denrées alimentaires au marché de gros de Bouaké. En effet, les acteurs étatiques sont soumis à des obligations de services publics délégués par l'Etat et délivre des documents facilitant le transport des produits vivriers. Quant aux acteurs non étatiques, ils répondent à un service dont les règles et l'exercice ont été édictés par la puissance publique, celui du transfert des produits vivriers vers les marchés.

Mots-clés : Bouaké, marché de gros, acteurs du transport, produits vivriers 


\title{
The Actors of the Transport of Food Products to the Wholesale Market of Bouake (Côte d'Ivoire)
}

\author{
Bamba Vakaramoko \\ Doctorant, Institut de Géographie Tropicale, \\ Université Félix Houphouët Boigny, Abidjan, Côte d'Ivoire \\ Koffié-Bikpo Céline Yolande \\ Professeur Titulaire, Institut de Géographie Tropicale, \\ Université Félix Houphouët Boigny, Abidjan, Côte d'Ivoire
}

\begin{abstract}
The supply chain of food products to the wholesale market of Bouaké presents peculiarities that are specific to the large African cities. It comprises of several actors on the entire chain, beginning from production to marketing, that entails the routing and the redistribution of products. The role played by these actors is fundamental to the supply of localities with food for their daily lives. This paper focuses on identifying the actors in the transport of food products and analyzing their role in the delivery of food products to the wholesale market of Bouaké.

Documentary research, the collection of data, their treatment, and the analysis of the results are the main joints of the methodological approach adopted. Based on the knowledge of the actors of the transport of food product to the wholesale market of Bouaké, the study has used the approach monitoring surveys of behavior to analyze the role of the actors in the refueling the wholesale market between the period 23 April 2018 to 05 May 2018. Additionally, 375 carriers have been investigated.

The results obtained show two types of actors: the state actors in the transport of food products and the non- State actors. This study highlights the importance of the actors of the transportation of foodstuffs to the wholesale market of Bouaké. Consequently, state actors are subject to the obligations of public services delegated by the State and they deliver documents which facilitates the transport of food products. With regard to non-State actors, they respond to a service whose rules and exercise have been enacted by public power. This, however, involves the transfer of food products to the markets.
\end{abstract}

Keywords: Bouake, wholesale market, actors in the transport, food products 


\section{Introduction}

Le marché de gros est caractérisé par sa dimension et la diversité de fonction qu'il assure. C'est le terminal du circuit d'approvisionnement et le début du reconditionnement pour la redistribution. Il est le lieu où s'organisent le stockage, la distribution et le transport vers d'autres horizons. Ces fonctions génèrent un grand nombre de flux qui eux-mêmes sont liés à d'autres activités. Le marché de gros joue un rôle important dans la compétition et le développement des lieux, des villes (Brunet et al, 1994, p. 318). Ainsi plusieurs acteurs assurent ces différentes relations. Les activités identifiées sur le marché (gros, demi gros et détail) et les catégories de produits vendus (produits frais et produits secs) supposent des flux distincts et des moyens de transport différents.

Quels sont les types d'acteurs du transport et comment ces acteurs acheminent les produits vivriers vers le marché de gros de Bouaké ? Le commerce de gros implique donc des entrepôts, des transitaires, des zones sous douane et demandent une gare routière. Tous ces types d'activités et de produits ont en commun de favoriser un trafic de véhicules, des petits porteurs jusqu'aux gros porteurs. Ce qui fait dire à Terpend et Kouyaté (1997, p.10) que les intervenants dans la commercialisation des denrées alimentaires sont nombreux en Afrique, avec une action directe ou indirecte sur la filière selon leurs activités. La présente étude vise alors à identifier ces acteurs et à analyser leur rôle dans l'acheminement des denrées alimentaires au marché de gros de Bouaké.

\section{I-Présentation de l'espace d'étude et approche méthodologique}

Dans cette partie, nous allons présenter notre cadre d'étude dans un premier temps et dans un deuxième temps donner l'approche méthodologique utilisée.

\section{I-1- Présentation de l'espace d'étude}

Le marché de gros de Bouaké (Figure 1) est une plate-forme de commercialisation des produits vivriers, unique en son genre dans la sousrégion ouest africaine. Il est situé à Bouaké, à $379 \mathrm{Km}$ de la capitale économique, Abidjan. Ce marché a une capacité d'accueil de 431 magasins dont 370 sont entièrement dédiés au vivrier. Il a été inauguré le 16 avril 1998 par le chef d'Etat ivoirien et son homologue nigérien pour montrer la symbolique d'un marché à vocation sous régionale. 


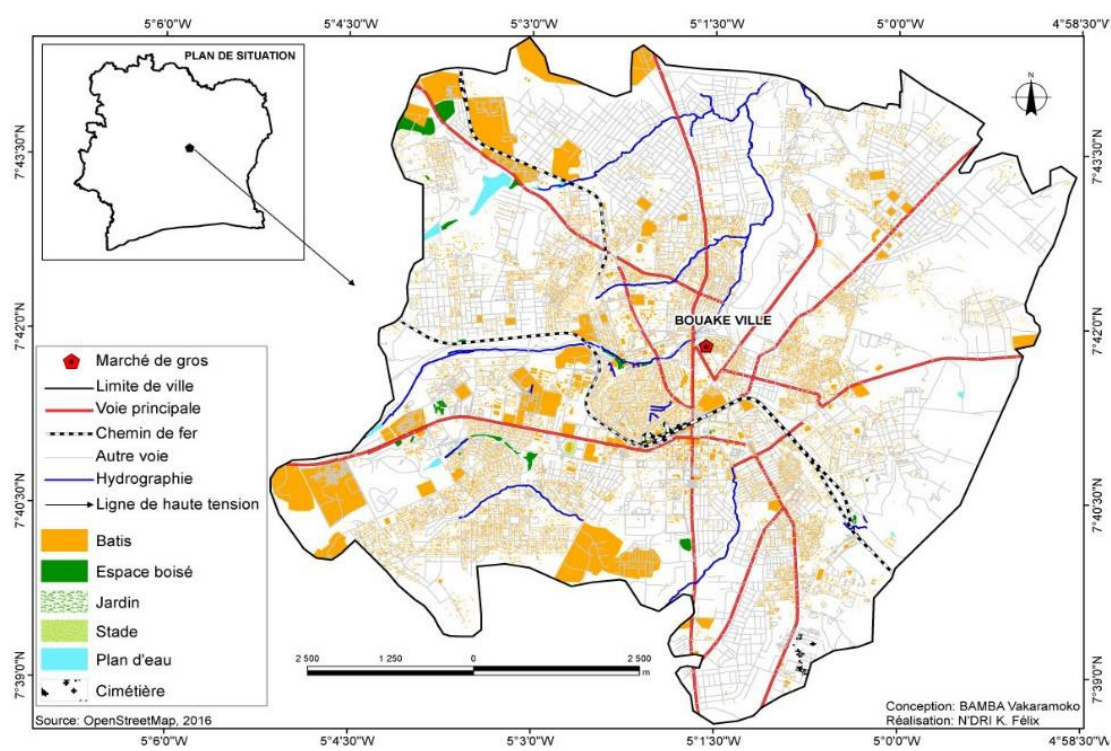

Figure 1. Localisation du marché de gros de Bouaké

\section{I-2- Approche méthodologique}

La méthodologie repose d'abord sur la recherche documentaire relative au sujet de recherche et ensuite la collecte des données primaires sur le terrain. La recherche documentaire a aidé à constituer la revue de la littérature des travaux effectués sur la redistribution et l'approvisionnement des produits vivriers. Ces ouvrages ont axé leurs analyses pour l'essentiel sur les circuits d'approvisionnement et de redistribution (Douka, 2011 ; Bikpo, 2015), le transport et les contraintes de transport (Kassi-Djodjo, 2011 ; Yéo, 2016). La collecte des données de terrain quant à elle s'est fondée sur l'approche des enquêtes de surveillance de comportement. Les transporteurs ont été enquêtés systématiquement quand ils viennent au marché de gros jusqu'à atteindre le nombre attendu dans chaque catégorie. La taille minimale de l'échantillon est obtenue à partir de la formule de Fisher suivante :

$$
n=\frac{t^{2} \times p(1-p)}{e^{2}}
$$

avec :

$\mathbf{n}=$ la taille d'échantillon requise, $\mathbf{t}=$ le niveau de confiance à $95 \%$ (valeur type de 1,96), $\mathbf{p}=$ la proportion des produits vivriers dans les produits entrant au marché de gros de Bouaké et $\mathbf{e}=$ la marge d'erreur à $5 \%$ (valeur type de 0,05). Les produits vivriers représentent $57,71 \%$ des produits enregistrés au marché de gros de Bouaké. Ainsi, nous avons :

$$
n=\overline{1,96^{i} 0,1^{2} 1(1-0,5771)}=375,04 \approx 375
$$


Ainsi nous avons enquêtés 375 véhicules sur les 1926 véhicules enregistrés (nos enquêtes, 2016) sur la période du 23 avril 2018 au 05 mai 2018.

\section{II-Résultats}

Les résultats obtenus se déclinent en deux parties : les acteurs étatiques du transport des produits vivriers et les acteurs non étatiques du transport au marché de gros de Bouaké.

\section{1-Les acteurs étatiques du transport des produits vivriers}

Les réseaux commerciaux et le facteur lié au transport déterminent l'articulation spatiale de l'approvisionnement du marché de gros. Le guichet unique des produits vivriers au marché de gros a pour objectif de regrouper tous les services pour faciliter toutes les formalités de déclaration. Il regroupe théoriquement le ministère de l'agriculture (certificat phytosanitaire), le ministère de l'industrie (certificat d'origine UEMOA), le ministère du commerce à travers l'OCPV (certificat de provenance), les eaux et forêt et la douane. Mais au marché de gros de Bouaké, cette structure fonctionne effectivement avec l'OCPV et la douane.

\section{1-1- L'OCPV, premier maillon du transport des produits vivriers}

L'Office d'aide à la Commercialisation des Produits Vivriers (OCPV) est un Etablissement Public National (EPN) à caractère administratif, sous tutelle technique du ministère du commerce et celui de l'économie et des finances. Il a pour mission d'apporter une aide multiforme aux opérateurs en vue d'une amélioration de la distribution et de la commercialisation des produits vivriers. La distribution est réservée à l'initiative privée selon l'article 4 du décret $\mathrm{N}^{\circ} 2012-961$ du 2 octobre 2012 selon le chapitre 1 portant création et attributions de l'OCPV. A ce titre, l'office est chargé d'étudier les problèmes relatifs à la collecte et à la distribution des produits vivriers, en vue de proposer toutes actions concrètes tendant à assurer une meilleure commercialisation de ces produits. Il contribue à l'organisation des marchés des produits vivriers, notamment de gros et de détail, en vue d'une meilleure performance du mécanisme et des infrastructures de ces marchés. Il participe à la définition et à l'application de toute politique visant à l'amélioration de l'approvisionnement et de la distribution des produits vivriers en Côte d'Ivoire. L'OCPV apporte son assistance aux commerçants des produits vivriers à travers la délivrance du certificat de provenance, et d'une manière générale favorise l'expansion du commerce du vivrier en Côte d'Ivoire. Le certificat de provenance créé par la loi des finances $\mathrm{N}^{\circ} 92-948$ du 23 décembre 1992 est destiné à faciliter les activités des commerçants de produits vivriers d'origine végétale, animale et halieutique. Ce certificat peut être considéré 
comme un titre de transport pour le transport des produits vivriers. Il vise trois (3) objectifs. Le premier est au niveau statistique. Il s'agit de capter les flux commerciaux des produits vivriers sur le territoire ivoirien ou à l'exportation. Le deuxième est économique, celui de présenter un laisser-passer aux différents postes de contrôle et de bénéficier de la priorité devant les autres chargements. Le dernier est financier, il s'agit de mettre en place un fonds de garantie pour soutenir les activités des acteurs du secteur du vivrier. C'est aussi un document administratif obligatoire délivré à tous les commerçants de produits vivriers. Le certificat est délivré sur les sites de chargement et de déchargement selon les coûts ci-après.

Tableau 1. Barème des montants à payer pour la délivrance d'un certificat de provenance

\begin{tabular}{|l|l|}
\hline Quantités de produits $(\mathrm{Kg})$ & Coût (FCFA) \\
\hline $0-1000$ & 500 \\
\hline $1001-5000$ & 1500 \\
\hline 5001 et plus & 3000 \\
\hline Import-Export & 5000 \\
\hline
\end{tabular}

Source : OCPV, Direction d'exploitation.

Le certificat de provenance est payé dans les agences de Caisse Nationale de Crédit et d'Epargne (CNCE) qui délivre un reçu. Il est aussi délivré dans les services de l'OCPV, sur les plates-formes de chargements de produits et, en l'absence de l'OCPV, par les agents du ministère de l'agriculture. Sous son nouveau mode opératoire, ce document se veut un instrument de relance économique. Il implique dans son exécution plusieurs entités que sont les forces de sécurité (Police, Douane, militaires), les transporteurs, les producteurs et les acteurs du vivrier. Tous se doivent de veiller à son bon fonctionnement.

La délivrance du certificat de provenance vise aussi un gain de temps et en efficacité lors des contrôles dans les corridors d'abord. Les montants payés pour s'en procurer varient de 500 à 5000 F CFA selon les quantités de produits acheminés. On assiste théoriquement à l'enrayement des faux-frais. Il apparaît comme un instrument efficace de lutte contre la cherté de la vie. Ensuite, les ressources générées par les entrées du certificat, permettront la création d'un fonds de garantie à l'endroit des acteurs du vivrier. Enfin, il permet de compter les flux commerciaux des produits vivriers que ce soit sur le plan local qu'international. Ces informations une fois traitées constitueront une base de données fiable selon l'OCPV.

Le transporteur quant lui, ne charge que des produits vivriers définis dans le certificat de provenance. Il doit avoir toutes les pièces administratives afférentes aux véhicules à jour et disposer d'un camion en bon état. Le certificat de provenance tel que décrit ne remplace pas les pièces du véhicule transportant les produits vivriers. C'est un document obligatoire, il est exigé aux corridors officiels par les forces de l'ordre (police, gendarmerie, douanes, 
eaux et forêts, FACI). Les agents de l'ordre ont le devoir de renvoyer les commerçants n'ayant pas le certificat de provenance sur le centre de délivrance le plus proche. L'acquisition de ce document se fait sur tous les sites de chargements des produits vivriers reconnus sur le territoire national. L'absence de certificat de provenance entraîne par contre une pénalité forfaitaire de $50000 \mathrm{~F}$ CFA payable avant tout déchargement. Mais ce certificat connait de nombreux problèmes entre autres son délai de validité, normalement de 3 jours renouvelables à partir de la date d'acquisition. En effet, cette validité est remise en cause après le troisième jour par les forces de l'ordre surtout au niveau des barrages non officiels. Aussi ce document peut être confié au transporteur en cas d'empêchement du commerçant, ce qui est souvent source de tracasseries routières.

\section{1-2- La douane : un acteur clé de l'importation et de l'exportation des produits vivriers}

Le marché de gros de Bouaké abrite un bureau annexe des douanes. Ce bureau est chargé de suivre les chargements et les déchargements des véhicules. Il est compétent pour l'enregistrement et le suivi de toutes les opérations d'exportation de marchandises. Il s'occupe de l'effectivité des exportations. Il est chargé du traitement des déclarations en détail des denrées du cru périssable. Il vérifie la conformité des chargements des marchandises. Le marché de gros est une plateforme à caractère national et international parce qu'il reçoit les produits vivriers du pays et les réexpédie dans les pays de la sous-région. Ainsi, deux (2) types de déclaration ont lieu au marché de gros. Ce sont la déclaration d'exportation des marchandises prises sur le marché intérieur (D6) et la déclaration de mise à la consommation directe de marchandises (D3). La D3 est un régime douanier de mise à la consommation directe des marchandises importées. Elle exige le paiement de droit et de taxes. Quant à la D6, c'est un régime douanier d'exportation simple, qui accompagne les marchandises qui sortent définitivement de la Côte d'Ivoire. Pour les produits vivriers, il n'y a pas de paiement de droit et taxes sauf pour le cas des produits tels que le café, le cacao, la noix de cola où il est perçu un Droit Unique de Sortie (DUS). Selon l'article 75 du code des douanes, il n'est pas perçu sur les produits vivriers un droit de douane. Le travail de la douane consiste en théorie, à une vérification de conformité entre la quittance et la déclaration. Les agents sont tenus de suivre l'établissement de la fiche de chargement. La déclaration en détail est un acte juridique par lequel le déclarant désigne d'abord le régime douanier dont il demande l'application aux marchandises concernées. Ensuite, il s'engage sous peine d'amende à accomplir les obligations du régime douanier déclaré, en l'occurrence les droits et taxes de la D3. Les procédures spécifiques sont exigées pour la déclaration de type D6, celle de l'exportation des produits vivriers pris sur le 
marché ivoirien (circulaire $\mathrm{N}^{\circ} 1298$ du 14 Novembre 2005). Les documents joints à la déclaration D6 sont d'une part des documents communs à tous les régimes c'est-à-dire la facture commerciale. D'autre part, les documents spécifiques comme l'engagement de change et l'attestation d'exportation sont ajoutés lorsque la marchandise à exporter à une valeur supérieure ou égale à 5 millions F CFA et que cette exportation a lieu en dehors de l'espace communautaire de l'UEMOA (journal officiel UEMOA, $\mathrm{N}^{\circ} 11 \mathrm{du}$ janvier 1999, article 13). En outre, il faut ajouter le certificat d'origine selon le circulaire $\mathrm{N}^{\circ} 1170$ du 25 janvier 2003 et le certificat phytosanitaire.

\section{2- Les acteurs non étatiques du transport et leurs modalités de travail au marché de gros de Bouaké}

Le secteur du transport au marché de gros met en relation de nombreux acteurs. Ce sont : les chauffeurs, les propriétaires, les syndicats, les coxers et les commerçants. Tous ces acteurs participent au bon ravitaillement en produits vivriers du marché de gros.

\section{2-1-Les syndicats, structure organisatrice du transport}

Il existe deux types de syndicats au marché de gros de Bouaké. Ce sont le syndicat des transporteurs et celui des chauffeurs. Il est parfois difficile de différencier le rôle de ces deux entités qui est presqu'identique. Ils veillent à la bonne distribution du fret entre les transporteurs.

L'organisation professionnelle des exploitants est assurée principalement par le Haut Conseil du Patronat des entreprises de transports routiers de Côte d'Ivoire. Elle a pour rôle la gestion de la station en théorie : conditions de chargement, ordre de départ des véhicules et les taxes (10 000 à 15000 F CFA indépendamment du tonnage), etc. En fait, le rôle essentiel et permanent du syndicat est un rôle d'intermédiation entre les opérateurs et les autorités, représentés sur le terrain par les forces de l'ordre. Il veille à ce que les véhicules ne soient pas contrôlés trop souvent, en instituant des relations cordiales avec les postes de contrôles concernés. C'est une des raisons de leurs créations. Les différents syndicats défendent les chauffeurs et les propriétaires en théorie, mais en pratique, ils préservent leurs propres intérêts. Le second rôle est la défense des intérêts des membres contre des exploitants extérieurs qui viendraient concurrencer sur le site. Ainsi des mesures de rétorsions sont souvent employées (destruction de véhicules, agression physique, etc.). Les syndicats développent parfois des activités sociales et d'entraide pour leurs membres en situation difficile: indisponibilité, accident, maladie, décès, mariage etc. Mais il faut ajouter que le travail des syndicats n'est pas exempt de reproche. Ils se livrent souvent à des rackets, des actes de vandalisme etc., au détriment de ceux qu'ils prétendent défendre. 


\section{2-2-Les transporteurs, acteurs indispensables à l'approvisionnement du marché de gros}

Les transporteurs interviennent comme des prestataires de services. Ils peuvent aussi être impliqués dans des opérations d'achat et de revente des produits vivriers. Pour la location du véhicule, le tarif varie en fonction de la distance, mais également en fonction de la situation géographique du lieu d'approvisionnement, du tonnage du véhicule et surtout en fonction de la période de l'année. Les transporteurs assurent le transport des marchandises des zones de production vers les grands centres urbains et les marchés de la sous-région. Ils se chargent de la collecte et/ou du transport des produits des lieux de collecte précédemment cités aux différents marchés de la ville. Ils sont aussi des intermédiaires à travers lesquels les commerçants grossistes ou non passent les commandes des marchandises qu'ils achètent dans les points appropriés. Ils sont soit des fournisseurs, soit des intermédiaires entre les fournisseurs situés dans les campagnes et les commerçants grossistes.

L'artisan transporteur est propriétaire d'un ou deux véhicules qu'ils exploitent pour le transport de marchandises. Les véhicules sont pour la plupart d'occasion, importé d'Europe, communément appelés « France au revoir ». Il exerce le transport à courte distance et le transport mixte. Néanmoins, il arrive qu'ils interviennent au niveau international également en fonction du gabarit du véhicule. Le personnel employé comprend généralement un chauffeur et un apprenti ou souvent deux selon le gabarit du véhicule. Le propriétaire traite avec les autorités locales ou nationales des questions des pièces afférentes au véhicule. Il négocie le fret auprès des commerçants, des syndicats ou des coxers en discutant le prix du transport. Il assure les frais de réparation du véhicule. Nos enquêtes montrent que 96,23\% des propriétaires sont responsable de l'entretien du véhicule. Ils n'ont pas de contrat permanent de réparation comme dans le cas des véhicules d'entreprise. $78,88 \%$ des enquêtés prétendent que leur employeur répare en cas de bonne trésorerie contre $22,03 \%$ en cas d'une sollicitation par un commerçant et $5,08 \%$ en cas d'un contrat de voyage. L'achat des pièces de rechange se fait généralement à la casse, soit $82,68 \%$ et chez un concessionnaire agréé, soit $17,32 \%$. La conséquence est qu'on retrouve des véhicules de transport de marchandises à la mécanique approximative sur les routes.

Le transport des produits vivriers au marché de gros est dominé par le secteur artisanal. L'entreprise artisanale est composée généralement par un propriétaire qui exploite ses propres véhicules. Il a recours à deux modes d'exploitation. Avec le premier mode, il assure la conduite et l'exploitation de son véhicule. Dans ce cas, il est appelé chauffeur-propriétaire. Dans le deuxième cas, il emploie des chauffeurs et un apprenti pour exploiter son véhicule. On note que $75,5 \%$ des chauffeurs n'ont pas de lien de parenté avec le propriétaire contre $24,5 \%$. 


\section{2-3-Les chauffeurs, cheville ouvrière du transport}

L'enquête sociodémographique des chauffeurs révèle que tous les acteurs du secteur sont des hommes. La majorité des enquêtés a un âge compris entre 21 et 35 ans soit $75,35 \%$ et $36,64 \%$ ont un âge qui oscille entre 36 et 70 ans. Ils sont à $92,9 \%$ d'origine ivoirienne. On y retrouve $70,7 \%$ de Malinké, $14,8 \%$ d'Akan, 9\% de Voltaïque, $09 \%$ de Krou et 4,6\% d'étrangers. La situation matrimoniale des acteurs présente $51,7 \%$ de célibataire, $41,8 \%$ de marié monogame et $6,5 \%$ de marié polygame. Les acteurs sont pour la plupart des analphabètes soit $43,6 \%, 26 \%$ ont fait l'école coranique, $20,8 \%$ le primaire, $8,7 \%$ le secondaire et $1 \%$ le supérieur.

Quant à l'exercice de l'activité, le chauffeur se voit confier la responsabilité d'un véhicule et travaille à plein temps. Il est responsable du véhicule devant son patron. Il est chargé de lui faire au quotidien le point sur l'état du véhicule et de verser à celui-ci la recette journalière. Il est l'interlocuteur direct du patron et est le seul salarié du véhicule. Les salaires des chauffeurs varient entre 40000 et 150000 F CFA par mois. En effet, 56\% des chauffeurs sont payés par mois, $24,8 \%$ le sont par voyage, 3,5\% par chargement et $15,6 \%$ le sont sur la base d'autres critères comme la ration journalière. Cette pratique consiste à faire la recette et le plein de carburant avant de se rémunérer soi-même.

Le chauffeur est aidé dans sa tâche par un apprenti. Celui-ci surveille l'état du camion et est tenu d'indiquer à son patron les entretiens et les réparations à effectuer sur le véhicule après chaque voyage. Il assure l'entretien courant et les petites réparations durant le voyage. Il négocie le prix $\mathrm{du}$ fret retour et est aussi responsable de la surveillance du véhicule. Il répare les crevaisons, vérifie également le chargement (le nombre de sacs). Il garde le véhicule pendant la nuit. Souvent les artisans ont recours à la main-d'œuvre familiale pour l'équipage du véhicule. Dans ce cas le chauffeur et son apprenti ont rarement une rémunération mensuelle. En période de traite, le chauffeur peut recevoir un salaire plus une prime journalière. Cette somme varie en fonction de la distance du voyage. De plus, l'employeur remet aux chauffeurs les frais de route (nourritures, contrôles routiers, carburant, divers...).

Le propriétaire et le chauffeur titulaire sont les seuls à convenir d'un accord. Cet accord concerne les conditions d'engagement du chauffeur et les modalités de travail. Généralement, pour engager un chauffeur dans le secteur artisanal, il n'existe pas de réglementation fixant les conditions d'embauche. Elles sont plutôt définies par les termes du contrat établi entre le propriétaire du véhicule et le chauffeur. Le contrat qui détermine les heures de travail, les dépenses et versements journaliers, mensuel, ou par voyage, par traite etc. se fait de façon verbale. Les conditions d'engagement sont définies et détaillées verbalement. Pour ce qui est du travail proprement dit, les deux entités 
définissent : l'embauche du chauffeur, la recette journalière et le salaire du chauffeur.

L'embauche d'un chauffeur sous-entend que le propriétaire lui confie la gestion de son véhicule. Il est le seul responsable du véhicule devant le propriétaire. Par conséquent, le chauffeur est chargé de lui faire le bilan journalier qui prend en compte la situation technique et financière du véhicule. Au plan financier, le chauffeur est tenu de rapporter, après la signature d'un contrat de transport, une somme qui varie selon le gabarit du véhicule. Sur le plan technique, le chauffeur s'occupe du véhicule durant le service et l'envoi au garage en cas de panne. Il faut noter que les petites pannes sont souvent à la charge du chauffeur, alors que la visite complète mensuelle ou semestrielle du véhicule est à la charge du propriétaire. Dans le cas du marché de gros, la rémunération mensuelle est la plus utilisée soit $56 \%$.

\section{2-4-Les coxers, intermédiaires entre transporteurs et commerçants}

Le coxer est un ancien chauffeur ou transporteur en général, qui a des entrées auprès des propriétaires et à une bonne expérience du métier. Généralement, il est très connu tant chez les commerçants que chez les transporteurs. Les coxers sont le plus souvent sollicités en période de récolte. Cette période correspond à une forte demande de véhicule, ce qui implique l'augmentation des coûts de transport. Ainsi le recours à un coxer peut être bénéfique quant à la négociation du coût du transport pour le commerçant. Le commerçant peut aussi négocier directement avec un transporteur pour le dépotage de sa marchandise, c'est la négociation directe. Cette transaction se fait généralement en période de pénurie. Cet arrangement prend effet quand le commerçant et le transporteur se côtoient. Dans ce cas un simple appel téléphonique suffit pour mettre le véhicule à la disposition du commerçant. Pour la recherche du fret le transporteur peut s'adresser directement aux chargeurs. Il peut utiliser les services d'un intermédiaire qui le mettra en contact avec un chargeur moyennant une commission variable selon la quantité de la marchandise et la distance. Celui-ci est de 5 à $10 \%$ du prix du transport. Les raisons qui concourent au recours à ces intermédiaires sont l'obtention de fret et le fait d'être une petite entreprise qui ne permet pas de discuter avec un grand nombre de commerçants. Plusieurs stratégies sont employées pour le transport des produits vivriers. La mobilisation d'un réseau de contact personnel et permanent c'est-à-dire la fidélisation de la clientèle qui suppose une bonne fiabilité et qualité de service des prestations de transport. Le recours au coxer, le système de tour de rôle et l'utilisation de sa propre flotte sont des gages pour une sollicitation de la part des commerçants. Les artisans transporteurs offrent une qualité de service souvent médiocre. Leurs véhicules sont en mauvais état et donc il y a des risques de pannes qui limitent leurs disponibilités. Les délais d'acheminement des marchandises sont 
allongés à cause des immobilisations administratives dues à l'état du véhicule (pneus lisses, forte surcharge), aux papiers non en règle et au fait que le personnel de conduite est insuffisamment formé.

\section{2-5-Les commerçants, usagers du service de transports au marché de gros}

$\mathrm{Ce}$ sont des commerçants régis par les articles 10 et 11 du décret $\mathrm{N}^{\circ} 99$ $445 \mathrm{du} 7$ juillet 1999. Ce décret stipule que ces commerçants sont des professionnels admis au marché de gros par une commission présidée par le préfet. Ils doivent vendre en gros. Pour la différenciation entre commerce de gros et de détail des produits vivriers fixés par les textes en vigueur, il est fait application de deux critères de sélection. Il y a le stock et la balance. Ils permettent de distinguer un commerce de gros et de détail selon la décision $\mathrm{N}^{\circ}$ 230 du 5 novembre 1999 de la direction du marché de gros. Cette décision s'appuie sur l'arrêté préfectoral $\mathrm{N}^{\circ} 153 \mathrm{~PB} / \mathrm{CAB}$ du 22 octobre 1999 portant organisation du commerce de gros de produits vivriers dans le périmètre de protection du marché de gros de Bouaké. Il est également considéré comme commerce de gros tout établissement ou commerçant possédant une balance d'une portée égale ou supérieure à $100 \mathrm{Kg}$ ou d'une balance de portée égale ou supérieure à $10 \mathrm{Kg}$ pour le cas particulier de l'oignon. Pour l'igname, il faut un stock de 100 tubercules.

Les grossistes achètent des stocks importants à des producteurs ou à des collecteurs soit en se déplaçant eux-mêmes vers les points d'approvisionnement, soit en traitant avec des fournisseurs localisés dans les points d'approvisionnement. Ils sont spécialisés dans un ou plusieurs produits de même catégorie, ceci en permanence. Il peut y avoir superposition des fonctions de grossiste et de détaillant, ou même de producteur et grossiste voire de détaillant. Les collecteurs-grossistes sont des commerçants ou intermédiaires. Ils achètent leurs produits auprès des producteurs aux champs ou dans les marchés ruraux, en vue de les revendre ultérieurement. Quand le grossiste ne se déplace pas lui-même, il confie cette tâche à un pisteur. Cela dépend du niveau de confiance entre les deux acteurs. Les commerçants confient leurs marchandises à des transporteurs avec qui, ils négocient généralement le tarif soit $28,7 \%$ des enquêtés. Mais les modalités de location les plus utilisés par les commerçants sont la location en fonction de la distance $(36,4 \%)$ et la location en fonction du poids $(28,7 \%)$. Le plus souvent une partie du coût de transport est payée avant le transport de la marchandise dans $52,5 \%$ des cas, contre $33,8 \%$ à l'arrivée de la marchandise et 13,8\% comme le payement différé sur quelques jours.

\section{III-Discussion}

Les acteurs étatiques et non étatiques participent à l'effectivité du transport des produits vivriers au marché de gros de Bouaké selon notre étude. 
Cette diversité d'acteurs dans l'approvisionnement du marché de gros est fondamentale dans les relations avec les zones de production proche et lointaine. Ainsi, le marché de gros devient le débouché majeur et permanent des produits vivriers. A ce propos, Vennetier (1991) affirme que «le propre d'une agglomération urbaine est d'établir avec un arrière-pays plus ou moins étendu, des liens multiples qui traduisent une complémentarité démographique et économique ». Ainsi les villes deviennent le lieu privilégié où s'exerce l'économie monétaire et par lequel il pénètre donc le monde rural, parce qu'elles font appel à ses productions ou se chargent de les collecter et de les commercialiser. Elles exercent une influence plus ou moins forte sur leur arrière-pays (Vennetier, 1991). Cela suppose une appréhension à la fois des infrastructures, des matériels, des différents groupes et des opérateurs intervenants au cours de diverses étapes de circulation et de stockage des produits. Le rôle des pouvoirs publics est donc déterminant dans la mise en lien au niveau du transport. Ils réglementent les infrastructures, l'organisation du marché, les transports et la gestion des entreprises de transport. Selon Mérenne (2013, p.203) «l'intervention de l'Etat s'effectue notamment via la législation et le financement des infrastructures ». Sur le plan local, régional et national, les pouvoirs publics sont responsables de la liberté de circulation, des produits, des populations et des moyens de transport. Le rôle de l'Etat est très important dans le fonctionnement du secteur des transports routiers des marchandises. La répartition des infrastructures, la hiérarchisation et la structuration des réseaux et des systèmes de transport, voire l'intensité des flux de circulation, peuvent être autant de choix opérés par les responsables politiques afin de promouvoir ou de maîtriser la demande. En définitive, l'Etat participe à divers stades de la gestion du domaine des transports à travers l'accès à la profession, l'organisation des transporteurs. Il participe donc diversement à la gestion du domaine des transports.

Il ressort de notre étude que le certificat de provenance délivré par l'OCPV pose souvent problème au niveau des barrages des forces de l'ordre comme le confirme l'étude de Yéo, (2016, p.54), pour qui «le certificat de provenance constitue un goulot d'étranglement pour la disponibilité, la stabilité et l'accessibilité des aliments sur les marchés de Korhogo ». L'Etat a mis en place l'office de la commercialisation des produits vivriers (OCPV), par le décret N84-934 du 27 juillet 1984 comme d'ailleurs dans plusieurs pays africains. Pour Chaléard (1996) "la création de l'office répond à la volonté de l'Etat de rester présent dans le négoce des vivres, sans pour autant être un acteur direct $»$.

Les résultats obtenus à partir de notre étude démontrent qu'il existe un lien étroit entre les différents acteurs non étatiques. L'étude montre une multitude d'acteurs qui part du syndicat jusqu'aux commerçants en passant par le transporteur comme l'ont démontré plusieurs études (Terpend \& 
Kouyaté, 1997 ; Hatcheu, 2006; Kassi.-Djodjo, 2011 ; Bikpo, 2015). En effet, l'élargissement des zones d'approvisionnement entraîne de facto une multiplication des agents le long du circuit d'approvisionnement. Ainsi, Terpend et Kouyaté (1997, p.10) confirment que « les transporteurs ont un rôle très important dans l'acheminement des produits ».

\section{CONCLUSION}

Les acteurs du transport des produits vivriers au marché de gros sont d'une part l'Etat qui participe diversement à la gestion du domaine des transports. Il choisit les infrastructures et édicte des lois pour réglementer l'usage du transport. Les institutions étatiques délivrent des documents qui permettent le transfert des produits vivriers au marché de gros et vers les autres marchés. Ainsi, le gouvernement à travers l'OCPV délivre des certificats de provenances et la douane quant à elle facilite l'obtention des déclarations douanières D3 et D6. Quant aux acteurs non étatiques, ils constituent l'épine dorsale du ravitaillement des marchés en Côte d'Ivoire en général et en particulier du marché de gros de Bouaké. Après le retrait de la puissance publique du négoce du vivrier, les acteurs non étatiques en l'occurrence les commerçants et les transporteurs ont envahis la chaîne du commerce du vivrier. En définitive, l'ensemble des acteurs de l'activité de transport du vivrier joue un rôle important dans l'approvisionnement du marché de gros.

\section{References:}

1. Bikpo Céline (2015). Politique agricole et approvisionnement de la ville d'Abidjan en produits vivriers locaux par les coopératives d'Abobo, Revue en ligne, RegardSuds, $\mathrm{N}^{\circ} 1$.

2. Brunet Roger, Ferras Robert \& Thery Hervé (1993). Les mots de la géographie. Dictionnaire critique $3^{\text {ème }}$ Ed, Montpellier/Paris, Reclus La documentation Française, $518 \mathrm{p}$.

3. Chaléard Jean Louis (1996). Temps des villes, temps des vivres. L'essor du vivrier marchand en Côte d'Ivoire, Paris, Karthala, 661p.

4. Douka Anne Marcelle (2011). La contribution des coopératives dans la régulation des marchés de vivriers à Abidjan in Production vivrière et sécurité alimentaire en Côte d'Ivoire, l'Harmattan Côte d'Ivoire, pp.93-108.

5. Godard Xavier (2002). Les transports et la ville en Afrique au sud du Sahara: le temps de la débrouille et du désordre inventif, Paris, Karthala-Inrets, 414p.

6. Hatcheu Emil Tchawe (2006). « Les commerçants et les transporteurs dans l'approvisionnement vivrier et la distribution alimentaire à Douala (Cameroun) », Bulletin de l'APAD [En ligne], 19|2000, mis 
en ligne le 12 juillet 2006, consulté le 30 décembre 2018. URL: http://journals.openedition.org/apad/431

7. Mérenne Emile (2013). Géographie des transports : contraintes et enjeux, Rennes, Presses Universitaires de Rennes, Edition révisée et actualisée 327p.

8. Kanga Koco Marie Jeanne (2013). Etude géographique des circuits de distribution et de commercialisation des produits vivriers dans l'approvisionnement d'Abidjan, Thèse de Doctorat Unique de géographie, Université FHB de Cocody, 284p.

9. Kassi-Djodjo Irène (2011). Transport et approvisionnement de la ville d'Abidjan en produits vivriers in Production vivrière et sécurité alimentaire en Côte d'Ivoire, l'Harmattan Côte d'Ivoire, pp.205-220.

10. Terpend Noëlle \& Kouyate Kalil (1997). Les contraintes des SADA des zones urbaines d'Afrique, le point de vue des acteurs des SADA, Dakar, FAO, Collection « Aliments dans les villes », 30p.

11. Vennetier Pierre (1991). Les villes d'Afrique tropicales, Paris, Masson, $244 p$.

12. Yéo Lanzeni, Coulibaly Tiécoura Hamed \& Koffié-Bikpo Céline Yolande (2016). Incidence du certificat de provenance des produits vivriers sur la sécurité alimentaire dans la ville de Korhogo, Abidjan, Educi, Revue de géographie tropicale et environnement, $\mathrm{N}^{\circ} 1$, pp.4655. 